\title{
Meat Hook Injury Leading to Brain Abscess: A Rare Occurrence
}

\author{
Batuk Diyora ${ }^{1}$ Gagan Dhall ${ }^{1} \quad$ Mehool Patel $^{1} \quad$ Mazharkhan Mulla ${ }^{1} \quad$ Nilesh More $^{1} \quad$ Amit Shah $^{2}$
}

${ }^{1}$ Department of Neurosurgery, Lokmanya Tilak Municipal General Hospital And Medical College, Mumbai, Maharashtra, India

${ }^{2}$ Criticare Hospital and Research Center, Mumbai, Maharashtra, India
Address for correspondence Batuk Diyora, DNB, Department of Neurosurgery, Second Floor, College Building, LTMG Hospital, Sion, Mumbai-22, Maharashtra, India (e-mail: bddiyora@gmail.com; batuk73@yahoo.co.in).

Indian J Neurotrauma 2022;19:111-116.

\begin{abstract}
Keywords

- intracerebral abscess

- transorbital injury

- intracranial penetrating injury

- meat hook

- occult transorbital injury

Transorbital orbitofrontal penetrating injury by a nonmissile object is uncommon. The presentation of this injury varies. This injury can be easily missed during the initial clinical presentation, because the foreign body is sometimes not visible on local examination, the wound on the orbital skin is small, and very subtle signs are present. The patient can present with delayed complications of the primary injury. Our patient was a 33-year-old male who presented with an orbitofrontal injury with a meat hook. He had minor symptoms at the time of presentation, which were overlooked. Three weeks later, he developed signs and symptoms of raised intracranial pressure (ICP). Brain imaging revealed a peripheral rim of contrast-enhancing mass lesion in the right frontal lobe, extending into the right orbit with perilesional edema suggestive of posttraumatic brain abscess. Via right frontal craniotomy, pus was drained out and abscess wall was excised. The patient made good clinical recovery. A higher index of suspicion and sound knowledge of occult penetrating injury patterns is required in the cases of orbital injuries. Appropriate radiological imaging can lead to an earlier and accurate diagnosis, and can prevent its delayed sequela like brain abscess.
\end{abstract}

\section{Introduction}

Penetrating orbital injuries is a significant threat to orbital and, often, cranial contents. Transorbital injuries represent up to $24 \%$ and $45 \%$ of all penetrating head injuries in adults and children, respectively. ${ }^{1}$ Transorbital orbitofrontal penetrating injury by a foreign body is infrequent, approximately $0.4 \%$ of all head injuries. ${ }^{2}$ It may present dramatically, with a severe large gaping wound or as subtle, innocuous, and initially occult injury. This occult injury may fail to be recognized initially, especially when the penetrating foreign object was removed, resulting in delayed presentation. Trivial-appearing eyelid lacerations may camouflage occult deep penetrations, which are frequently sutured without further investigation. ${ }^{3}$ This occult injury is more dangerous, as it can be easily missed

published online July 29, 2021
DOI https://doi.org/ $10.1055 / \mathrm{s}-0041-1732789$ ISSN 0973-0508 and may present later with potentially life-threatening complications. ${ }^{4,5}$ In this article, a case of a meat hook causing occult transorbital orbitofrontal penetrating injury, resulting in brain abscess was described and literature reviewed.

\section{Case Report}

A 33-year-old male patient had suffered atypical transorbital penetrating injury when he suddenly lost his balance due to a slip while standing in the meat shop in front of meat hooks and his head accidentally plunged into the sharp/pointed arm of an S-shaped bare meat suspension hook. The meat hook penetrated his orbit through a small cut over his right eyebrow, and he immediately experienced severe pain.

(C) 2021. Neurotrauma Society of India.

This is an open access article published by Thieme under the terms of the Creative Commons Attribution-NonDerivative-NonCommercial-License, permitting copying and reproduction so long as the original work is given appropriate credit. Contents may not be used for commercial purposes, or adapted, remixed, transformed or built upon. (https://creativecommons.org/licenses/by-nc-nd/4.0/).

Thieme Medical and Scientific Publishers Pvt. Ltd. A-12, 2nd Floor, Sector 2, Noida-201301 UP, India 
He grabbed the hook with his hands, balanced and adjusted himself, and gently pulled out themeathookonhisown. Hetook an analgesic medication and did not pay much attention to it. The next morning, he discovered swelling in his right eyelid and diffuse redness in his right eye, so he consulted an ophthalmologist who prescribed him oral analgesic and antibiotic eyedrops and oral antibiotics for 5 days. Within the next 3 to 4 days, he had no pain, redness in his right eye disappeared, and the eyelid swelling reduced. He had no further consultation. Three weeks after his initial injury, he presented with severe progressive holocranial, dull-aching, continuous headache for 2 to 3 days along with giddiness. On examination, he was drowsy but easily arousable. His Glasgow coma scale (GCS) score was approximately 14/15 (E3M6V5). Plantars were extensor on the left side and flexor on right side. Ophthalmological examination revealed right upper eyelid swelling and right conjunctival congestion. Both pupils were of normal size, isochoric, and reacting normally to light. His visual acuity and extraocular movements were normal. He had a small, healed wound over the right eyelid (-Fig. 1). He received a tetanus toxoid injection along with intravenous (IV) antibiotics and antiepileptic medications. The CT scan brain revealed right frontal lobe edema. CT scan of orbit revealed minimally displaced fracture of the roof of the right orbit with mild angulation of the fractured fragment and periorbital edema. Contrast-enhanced CT scan of the brain and orbit revealed a peripheral irregular ring-enhancing lesion of approximately $3.1 \times 4.4 \times$ $5.3 \mathrm{~cm}$ size in the right frontal lobe with perilesional edema. The lesion was communicating with the orbit through a bony defect in the orbital roof ( $\mathbf{- F i g}$. 2A, 2B, 2C, 2D). MRI of the brain with orbit revealed $3.2 \times 4.6 \times 5.2 \mathrm{~cm}$ size peripheral rim-enhancing mass lesion over right frontal lobe, communicating with an orbit which was isointense on T1-weighted images and hyperintense on T2-weighted image with moderate perifocal vasogenic edema ( - Fig. 3A, 3B, 3C, 3D).

The patient underwent a right frontal craniotomy. The dura mater was tense and brain wall full. Via the anterior part of the middle frontal gyrus, a lesion entered. Brownish,

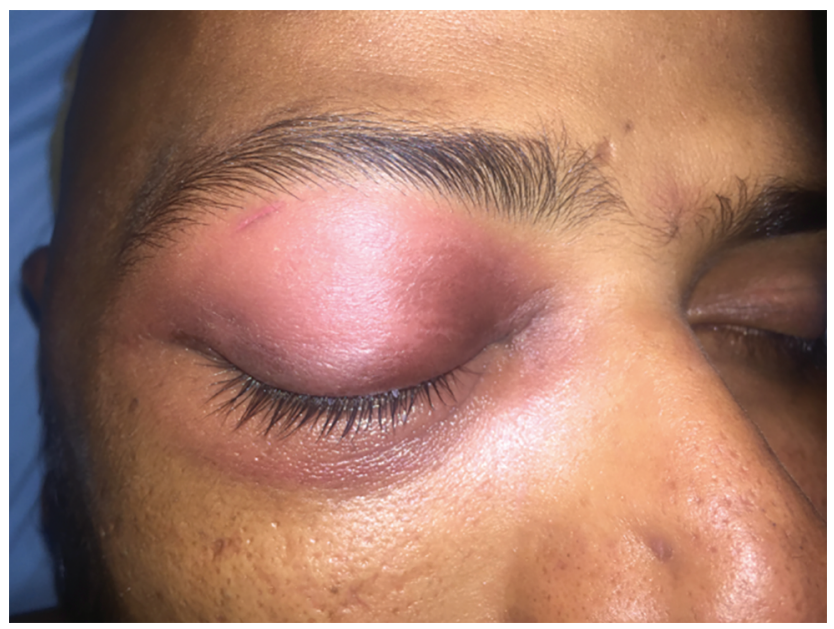

Fig. 1 Clinical photograph showing right eyelid edema, redness and small healed punctured wound over upper eyelid just below lateral one third of right eyebrow. viscous, nonfoul-smelling pus of approximately $30 \mathrm{ml}$ aspirated and cyst wall excised. Subsequently, the brain became lax. Part of the frontal lobe just above the anterior part of

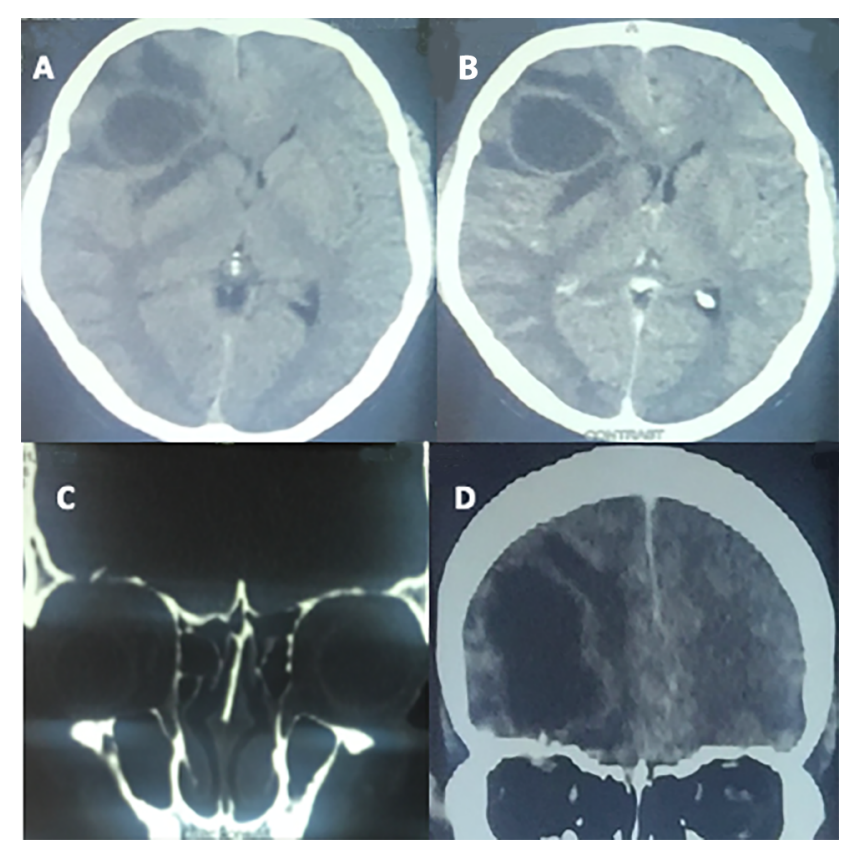

Fig. 2 CT scan of brain showing right frontal cystic lesion with perilesional edema (A). Lesion was enhancing after intravenous (IV) contrast administration (B). CT scan brain and orbit, coronal view showing cystic cavity just above fractured orbital roof (D), and bone window showing right orbital roof fracture (C).

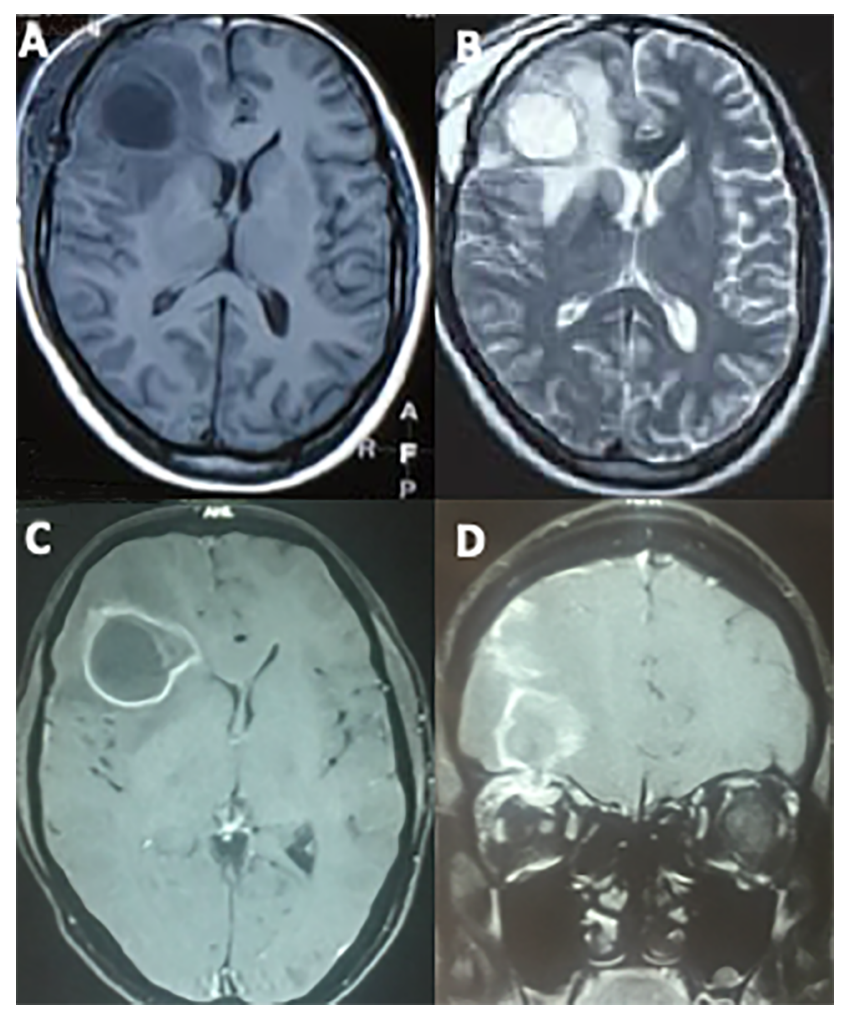

Fig. $3 \mathrm{MRI}$ of brain showing right frontal cystic lesion on axial T1-weighted image $(\mathbf{A})$ and T2-weighted image (B) with perilesional edema with mass effect. Lesion showing peripheral rim of contrast enhancement $(\mathbf{C})$. Postcontrast coronal view image showing right frontal contrast-enhancing lesion communicating with orbit (D). 
the orbital roof was adherent to it. On gentle separation of frontal lobe from the orbital roof, a $15 \times 8 \mathrm{~mm}$ size defect was found in the basal frontal dura and orbital roof. Two free bony fragments adjacent and superior to the orbital roof were also found along with granulation tissue and pus. Free-floating bony fragments, granulation tissue, along with the pus, were removed and cavities thoroughly irrigated with antibiotic solutions. The dural defect over the orbital roof was closed with a temporalis fascia graft.

The postoperative course was uneventful. Headache completely disappeared. Right eyelid swelling also subsided within 4 to 5 days. The patient was empirically started on broad-spectrum antibiotics (IV vancomycin, cephalosporin, and metronidazole). He also received antiepileptic (phenytoin) in the postoperative period. Postoperative contrast-enhanced CT scan revealed no residue of the abscess, disappearance of ring-like enhancement and decreased brain edema. The microbial culture of the pus from the brain abscess grew colonies of Staphylococcus aureus, sensitive to vancomycin. The patient was discharged on the seventh postoperative day without any neurological deficit. He received IV antibiotics for 2 weeks and oral antibiotics for 4 weeks. He received oral anticonvulsants for 3 months.

At 3 months follow-up, the patient was completely asymptomatic. His visual acuity and fields of vision were completely normal. Follow-up MRI images revealed complete resolution of an abscess on postgadolinium administration (- Fig. 4A, 4B, 4C, 4D).

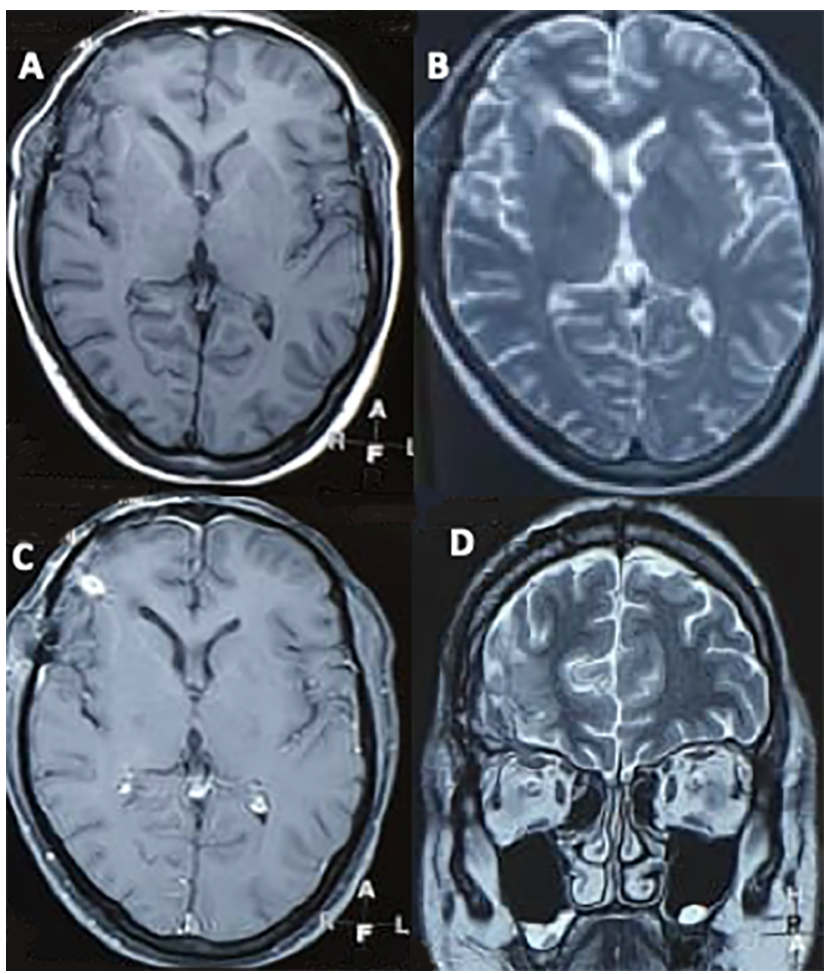

Fig. $4 \mathrm{MRI}$ of brain showing complete resolution of right frontal lesion on T1-weighted axial mage (A), T2-weighted axial (B) and coronal image (D). Very subtle enhancement was seen after intravenous (IV) contrast administration (C).

\section{Discussion}

The bony cranium can be entered through the orbit via its weak walls. The most common route of entry is a thin orbital roof. ${ }^{1,}$ Superior orbital fissure is the second most frequent site through which foreign bodies occasionally reach the brain stem via cavernous sinus, resulting in a severe injury. ${ }^{6}$ A third, rarer avenue of penetration is the optic canal, where the object is directed into the suprasellar cistern, close to the optic nerve and internal carotid artery. ${ }^{7}$ Turbin and colleagues analyzed transorbital intracranial injury patterns and divided the orbital surface into different zones. ${ }^{1}$ Balasubramanian and colleagues classified transorbital penetrating injury, based on the orbital bone's anatomy and the associated injury. ${ }^{8}$ This analysis of injury patterns could help one tailor the management and surgical approach, and anticipate the potential type and sites of intracranial complications related to foreign body penetration.

Orbital injuries can cause vision loss by damaging the orbital contents or optic nerve. Bilateral vision loss in unilateral orbital injury due to direct chiasmal and indirect ischemic vascular injuries has also been described. ${ }^{9}$ Penetrating orbital injury gives rise to severe brain injury when the foreign object enters the cranium, leading to both orbital and cerebral complications. Because of the small entry point, incomplete history, and trivial nature of the injury, caregivers easily overlook it. ${ }^{1}$ In such cases, eyelid laceration is sutured without further investigation and these patients present with delayed complications like rhinorrhea, orbital cellulitis, eyelid abscess, cerebritis, subdural empyema, recurrent meningitis, encephalitis, cerebral and intraorbital abscesses, pseudoaneurysm, and posttraumatic arteriovenous malformation. ${ }^{4,10,11}$

Brain abscess following transorbital orbitofrontal injuries is rare. In literature review, we found about 10 cases. ${ }^{12-21}$ - Table 1 summarizes the studies of transorbital craniocerebral penetrating injuries complicated by an intracerebral abscess.

The brain abscesses may result from either the foreign body's presence or the bone fragments from a skull fracture that have been driven further into the cranial tissue. Organisms present on the surface of foreign objects and bacteria present on the skin at the site of impact can be transported not only to the orbit but also to the cranium when there is an orbital roof fracture. Therefore, such an injury may cause severe infectious complications from days to years after the initial injury. Following transorbital transcranial injury, the patient can develop eyelid abscess within 3 weeks and brain abscess within a few weeks to months. ${ }^{14}$ Formation of brain abscess runs through four radiological and histopathological stages: early cerebritis (1-4 days), late cerebritis (4-10 days), early capsule formation (11-14 days), and late capsule formation (> 14 days). ${ }^{22}$ Early cerebritis is an early stage of infection with coagulative necrosis. It progresses to late cerebritis with mainly liquefactive type of necrosis. Cerebritis may progress and organize to form an abscess. Early capsule stage has a very thin capsule of granulation tissue around a central necrotic material. It appears as a discrete lesion with thin enhancing rim on imaging. 


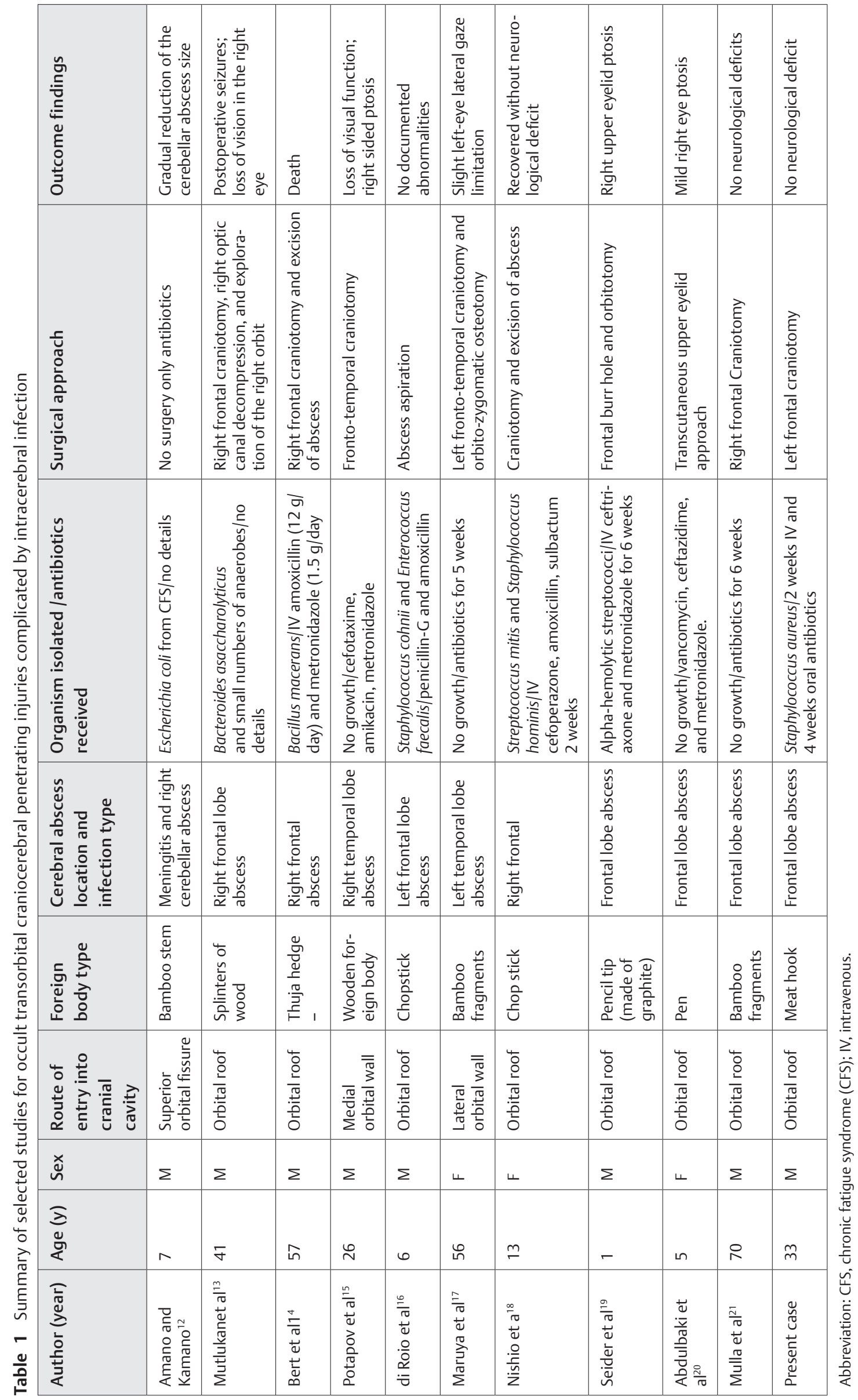


Late encapsulation is a process where the central necrosis progresses further, the cavity shrinks, and edema decreases. In our case, the patient presented with brain abscess symptoms after 21 days of the injury. Brain abscess occurs in $50 \%$ of intracranial injuries following a periorbital wound. ${ }^{23}$ The duration required for brain abscess development depends on the potency, infectivity, and virulence of a particular organism. ${ }^{24}$ In our case, the patient developed brain abscess, possibly due to direct implantation of the infecting organism (S. aureus based on microbial cultures) by the meat hook, although there was no retained foreign body in the orbit or cranium.

The management of penetrating transorbital craniocerebral head injuries depends on the presence/absence and foreign body location, its tract in the orbit and cranium, associated bony or neural injuries, intracranial complications, and the time interval between initial injury and treatment. ${ }^{1,21}$ The clinical goals of such operations include preventing infection, decreasing the possibility of epilepsy (by giving antiepileptic medications), controlling bleeding from affected cerebral vessels, and preventing cerebrospinal fluid (CSF) fistula and brain abscess.

Initial empirical antimicrobial therapy should be based on the suspected organisms, underlying predisposing conditions, and expected source of infection. Broad-spectrum parenteral antibiotics which can cross blood-brain barrier (BBB) and blood-CSF barrier, and can accumulate in the abscess cavity in bactericidal concentrations, are chosen. Cephalosporins are the most preferred antibiotics. ${ }^{9}$ In abscesses due to trauma or neurosurgical procedures, combination of the vancomycin, third- or fourth-generation cephalosporin and metronidazole is recommended. ${ }^{25}$ In immunosuppressed patients, therapies for Nocardia (trimethoprim-sulfamethoxazole), Aspergillus (voriconazole), or Toxoplasma (pyrimethamine-sulfadiazine) can be added. ${ }^{26}$ Once the pus is drained and the antibiotic culture and sensitivity report is available, patients are started on specific bactericidal agents. If the culture is negative for any organism, the empirically given broad-spectrum antibiotics can be continued. According to the British Society for Antimicrobial Chemotherapy, only 1 to 2 weeks of IV therapy is recommended. ${ }^{27}$ The recommended duration of IV antimicrobial therapy in other studies varies widely from 2 weeks to 8 weeks. Dattatraya et al suggested "triple high dose" antibiotics IV for 2 weeks, followed by four weeks of oral therapy.22 Agrawal et al recommend appropriate IV antibiotics administered for 3 weeks, followed by oral antibiotics for another 3 weeks. ${ }^{28}$ Some suggest IV antibiotic therapy for 6 to 8 weeks, followed by a course of an oral agent. Common oral treatment regimens include ciprofloxacin, metronidazole, and amoxicillin. In general, antibiotic treatment is generally advised as long as the abscess cavity is visible on cranial MRI. ${ }^{26}$ Intraventricular antimicrobials are recommended after intraventricular rupture of brain abscess. Opportunistic organisms may need longer, 3 to 12 months, antibiotic courses. ${ }^{22}$ Surgical treatment includes evacuation of the cavity by aspiration of its contents, debridement of the path, excision of the abscess wall, removing the foreign body, and any retained bone fragments. ${ }^{18,29,30}$

\section{Conclusions}

Transorbital transcranial penetrating injuries are uncommon injuries and can be life-threatening if not diagnosed and treated appropriately on time. These injuries can easily be missed when periorbital swelling is present and the foreign body is not visible outside. Appropriate radiological imaging can lead to an earlier and accurate diagnosis and prevent delayed sequelae like brain abscess.

\section{Funding \\ None.}

\section{Conflict of Interest}

None declared.

\section{References}

1 Turbin RE, Maxwell DN, Langer PD, et al. Patterns of transorbital intracranial injury: a review and comparison of occult and non-occult cases. Surv Ophthalmol 2006;51(5):449-460

2 Satyarthee GD, Dawar P, Borkar SA, Sharma BS. Trans-orbital penetrating head injury (TOPHI): Short series of two cases with review of the literature. Indian J Neurotrauma 2014;11:49-52

3 Guthkelch AN. Apparently trivial wounds of the eyelids with intracranial damage. BMJ 1960;2(5202):842-844

4 Bard LA, Jarrett WH. Intracranial complications of penetrating orbital injuries. Arch Ophthalmol 1964;71:332-343

5 De Villiers JC, Sevel D. Intracranial complications of transorbital stab wounds. Br J Ophthalmol 1975;59(1):52-56

6 Shin TH, Kim JH, Kwak KW, Kim SH. Transorbital penetrating intracranial injury by a chopstick. J Korean Neurosurg Soc 2012;52(4):414-416

7 Matsumoto S, Hasuo K, Mizushima A, et al. Intracranial penetrating injuries via the optic canal. AJNR Am J Neuroradiol 1998;19(6):1163-1165

8 Balasubramanian C, Kaliaperumal C, Jadun CK, Dias PS. Transorbital intracranial penetrating injury-an anatomical classification. Surg Neurol 2009;71(2):238-240

9 Singh MK, Deora H, Tripathi M, Mohindra S, Batish A. Penetrating injury of the eye causing bilateral visual loss: an eye opener! Asian J Neurosurg 2019;14(3):943-945

10 Duffy GP, Bhandari YS. Intracranial complications following transorbital penetrating injuries. Br J Surg 1969;56(9):685-688

11 Kazarian EL, Stokes NA, Flynn JT. The orbital puncture wound: intracranial complications of a retained foreign body. J Pediatr Ophthalmol Strabismus 1980;17(4):247-250

12 Amano K, Kamano S. Cerebellar abscess due to penetrating orbital wound. J Comput Assist Tomogr 1982;6(6):1163-1166

13 Mutlukan E, Fleck BW, Cullen JF, Whittle IR. Case of penetrating orbitocranial injury caused by wood. $\mathrm{Br}$ J Ophthalmol 1991;75(6):374-376

14 Bert F, Ouahes O, Lambert-Zechovsky N. Brain abscess due to Bacillus macerans following a penetrating periorbital injury. J Clin Microbiol 1995;33(7):1950-1953

15 Potapov AA, Eropkin SV, Kornienko VN, et al. Late diagnosis and removal of a large wooden foreign body in the cranio-orbital region. J Craniofac Surg 1996;7(4):311-314

16 Di Roio C, Jourdan C, Mottolese C, Convert J, Artru F. Craniocerebral injury resulting from transorbital stick penetration in children. Childs Nerv Syst 2000;16(8):503-506, discussion 507

17 Maruya J, Yamamoto K, Wakai M, Kaneko U. Brainabscess following transorbital penetrating injury due to bamboo fragmentscase report. Neurol Med Chir (Tokyo 2002;42(3):143-146 
18 Nishio Y, Hayashi N, Hamada H, Hirashima Y, Endo S. A case of delayed brain abscess due to a retained intracranial wooden foreign body: a case report and review of the last 20 years. Acta Neurochir (Wien 2004;146(8):847-850

19 Seider N, Gilboa M, Lautman E, Miller B. Delayed presentation of orbito-cerebral abscess caused by pencil-tip injury. Ophthal Plast Reconstr Surg 2006;22(4):316-317

20 Abdulbaki A, Al-Otaibi F, Almalki A, Alohaly N, Baeesa S. Transorbital craniocerebral occult penetrating injury with cerebral abscess complication. Case Rep Ophthalmol Med 2012;2012:742186

21 Mulla M, Bhende B, Patil S, Patil A. Diyora B. Civilian occult orbitocranial penetrating injury presenting with a cerebral abscess in an elderly patient. Indian JNeurotrauma 2017;14(2):145-148

22 Muzumdar D, Jhawar S, Goel A. Brain abscess: an overview. Int J Surg 2011;9(2):136-144

23 Miller CF, Brodkey JS, Colombi BJ. The danger of intracranial wood. Surg Neurol 1977;7(2):95-103

24 Brook I. Brain abscess in children: microbiology and management. J Child Neurol 1995;10(4):283-288
25 Brook I. Microbiology and treatment of brain abscess. J Clin Neurosci 2017;38:8-12

26 Brouwer MC, van de Beek D. Epidemiology, diagnosis, and treatment of brain abscesses. Curr Opin Infect Dis 2017;30(1):129-134

27 Infection in Neurosurgery Working Party of the British Society for Antimicrobial Chemotherapy. The rational use of antibiotics in the treatment of brain abscess. $\mathrm{Br}$ J Neurosurg 2000;14(6):525-530

28 Agrawal D, Suri A, Mahapatra AK. Primary excision of pediatric posterior fossa abscesses-towards zero mortality? A series of nine cases and review. Pediatr Neurosurg 2003;38(2):63-67

29 Kuntzman J. Meningitis following perforation of the roof of the orbit by a pencil. Strasbourg Med J 1938;98:154

30 Liu WK, Ma L, Mao BY. A delayed frontorbital abscess caused by a penetrating nonmissile foreign body (a bamboo stick) Neurol India 2009;57(2):208-210 